\title{
EVALUACIÓN BIOLÓGICAEN RATAS DE LABORATORIO (Rattus norvegicus) DE FUENTES PROTEICAS USADAS EN ALIMENTOS COMERCIALES PARA PERROS
}

\author{
Walter Silva S. ${ }^{1}$, Teresa Arbaiza F. ${ }^{2}$, Fernando Carcelén C. ${ }^{2}$ y Orlando Lucas A. ${ }^{2}$
}

\section{ABSTRACT}

The aim of this study was to biologically assess three protein sources used in commercial dog food: soy meal (TS), meat meal (HC), and chicken meal (HP) using four biological tests: Protein Efficiency Ratio (PER), True Digestibility (DV), Net Protein Utilization (NPU) and Biological Value (VB). 40 albino laboratory rats of 23 days of age were used. Five groups were formed according to the protein source: groups TS, HC, HP, control group (sodium caseinate) and a non protein-group. The results were: PER: TS = $1.86, \mathrm{HC}=1.39, \mathrm{HP}=1.69$, control $=2.11 ; \mathrm{DV}: \mathrm{TS}=98.76, \mathrm{HC}=96.95, \mathrm{HP}=98.13$, control =99.19; $\mathrm{NPU}: \mathrm{TS}=58.19, \mathrm{HC}=35.52, \mathrm{HP}=60.19$, control $=96.46 ; \mathrm{VB}: \mathrm{TS}=0.59, \mathrm{HC}=0.37$, $\mathrm{HP}=0.61$, control $=0.97$. PER results in the $\mathrm{HC}$ group were significantly lower than other groups $(\mathrm{p}<0.05)$. There was not statistical differences on PER between groups HP and TS. All protein sources had a high true digestibility. The HC was the protein source of less quality because the lowest PER, NPU and VB values. It is concluded that to asses the quality of protein nutrients is not enough to establish the protein content and digestibility, but to conduct biological tests.

Key words: soy meal, meat meal, chicken meal, PER, NPU, digestibility, biological value, rats

\section{RESUMIEN}

El objetivo del estudio fue realizar una evaluación biológica a tres insumos proteicos utilizados en la alimentación comercial de perros: torta de soya (TS), harina de carne (HC) y harina de pollo (HP). Se utilizaron las pruebas biológicas de Relación de Eficiencia Proteica (PER), Digestibilidad Verdadera (DV), Utilización Neta de las Proteínas (NPU) y Valor Biológico (VB). Se empleó 40 ratas albinas de 23 días de edad distribuidas en grupos de acuerdo al insumo proteico utilizado en la dieta: grupos TS, HC, HP, control (caseinato de sodio) y aproteico. Los resultados obtenidos fueron: PER: TS $=1.86, \mathrm{HC}=$ 1.39, $\mathrm{HP}=1.69$, control = 2.11; $\mathrm{DV}: \mathrm{TS}=98.76, \mathrm{HC}=96.95, \mathrm{HP}=98.13$, control = 99.19; NPU: $\mathrm{TS}=58.19, \mathrm{HC}=35.52, \mathrm{HP}=60.19$, control $=96.46$; $\mathrm{VB}: \mathrm{TS}=0.59, \mathrm{HC}=0.37, \mathrm{HP}=$ 0.61, control $=0.97$. La PER de la HC fue significativamente menor que la PER de los otros insumos $(\mathrm{p}<0.05)$. No hubo diferencias estadísticas entre la PER de la HP y TS. Los insumos proteicos evaluados tuvieron una alta DV. La HC es el insumo proteico de menor calidad (menor PER, NPU y VB). Se concluye que para evaluar la calidad de los insumos proteicos no es suficiente determinar su contenido proteico y digestibilidad, sino que además se requiere realizar las evaluaciones biológicas.

Palabras clave: torta de soya, harina de carne, harina de pollo, PER, NPU, digestibilidad, valor biológico, ratas

${ }^{1}$ Práctica privada

${ }^{2}$ Laboratorio de Bioquímica, Nutrición y Alimentación Animal, FMV-UNMSM 


\section{INTRODUCCIÓN}

El perro doméstico (Canis familiaris) es un carnívoro que se ha adaptado al consumo de una gran variedad de insumos en su alimentación. Dentro de los insumos proteicos de origen animal usados en la alimentación comercial de perros se tiene la carne bovina, ovina, de pollo y de conejo, así como de sus subproductos, además de huevo deshidratado, pescado y harina de pescado, entre otros (Church y Pond, 1990; Case et al., 1997). La calidad de la harina de carne varía considerablemente de acuerdo a la calidad de los residuos que la componen (músculo, tejido conjuntivo o cartilaginoso, sangre, huesos). Una harina de carne en buenas condiciones contiene 65\% de proteínas (Piccioni, 1970). Por otro lado, los tratamientos térmicos muy elevados, así como los tratamientos químicos pueden desnaturalizar las proteínas, reduciendo su digestibilidad y la fracción útil de lisina.

La harina de pollo está compuesta de restos de aves no consumibles por el hombre, además de plumas, uñas, buche, intestinos y huesos. Las plumas tienen un $87 \%$ de proteínas, pero son de baja digestibilidad, aunque la calidad puede ser mejorada por medios físicos o químicos (Fataccioli, 1981).

En cuanto a las fuentes proteicas de origen vegetal se encuentran la harina de gluten de maíz, torta de soya, harina de alfalfa, levadura de cerveza seca, harina de semillas de linaza y el germen de trigo (Church y Pond, 1990; Case et al., 1997). La soya es un insumo empleado en la nutrición de varias especies de interés económico para el hombre. Piccioni (1970) menciona un porcentaje proteico para la torta de soya de 44 a $46 \%$. El calentado de la soya o tostación resulta indispensable para que pueda exhibir todas sus ventajas ya que en forma natural contiene diversos principios tóxicos incluyendo un factor deprimente del crecimiento.

Existen varios métodos para evaluar su calidad proteica, y están relacionados con la eficiencia con la que son usados para la síntesis y mantenimiento de la proteína tisular. Los métodos biológicos se fundamentan en el crecimiento de ratas destetadas. La aplicación de los valores de los ensayos biológicos en ratas con respecto a la nutrición proteica en perros es mayormente de tipo cualitativo.

En nuestro país existe una variedad de marcas de alimentos para perros, tanto nacionales como importados con diferentes tipos de insumos e indudablemente con diferentes calidades proteicas. El objetivo del presente estudio fue evaluar a través de pruebas biológicas en ratas el valor nutricional de los insumos proteicos utilizados en los alimentos comerciales para perros.

\section{Materiales y Métodos}

\section{Generalidades}

El estudio se realizó en el bioterio y en el Laboratorio de Bioquímica, Nutrición y Alimentación Animal de la Facultad de Medicina Veterinaria, Universidad Nacional Mayor de San Marcos. Se empleó 40 ratas albinas (Rattus norvegicus) de 23 días de edad.

Se evaluó 5 dietas, las que se denominaron de acuerdo al insumo proteico: TS (torta de soya), HC (harina de carne), HP (harina de pollo), control (caseinato de sodio) y aproteico (Cuadro 1). El criterio de formulación se basó en el método oficial de la AOAC (1990), donde se especifica que las raciones deben contener un $10 \%$ de proteína. Las raciones fueron molidas y tamizadas a través de una malla de $1 \mathrm{~mm}$. Además del insumo proteico, las dietas contenían aceite de maíz, celulosa, almidón de maíz, agua, así como mezclas de vitaminas y minerales.

Los insumos proteicos utilizados en el presente trabajo se obtuvieron de una empresa nacional que prepara alimentos balanceados para perros; sin embargo, los alimen- 
Cuadro 1. Composición porcentual de las $\operatorname{dietas}^{1}$ utilizadas en las pruebas biológicas $(\mathrm{g} / 100 \mathrm{~g})$

\begin{tabular}{lccccc}
\hline & $\begin{array}{c}\text { Dieta TS } \\
(\%)\end{array}$ & $\begin{array}{c}\text { Dieta HC } \\
(\%)\end{array}$ & $\begin{array}{c}\text { Dieta HP } \\
(\%)\end{array}$ & $\begin{array}{c}\text { Control } \\
(\%)\end{array}$ & $\begin{array}{c}\text { Aproteica } \\
(\%)\end{array}$ \\
\hline Torta de soya & 21.36 & ----- & ---- & ----- & ----- \\
Harina de carne & ---- & 23.12 & ---- & ---- & ---- \\
Harina de pollo & ---- & --- & 20.17 & ---- & ---- \\
Caseína & ---- & ---- & ---- & 16.00 & ---- \\
Aceite de maíz & 7.57 & 5.82 & 2.57 & 10.00 & 10.00 \\
Mezcla de minerales & 3.91 & 3.76 & 1.95 & 4.00 & 4.00 \\
Mezcla de vitaminas & 1.00 & 1.00 & 1.00 & 1.00 & 1.00 \\
Celulosa & 0.16 & 1.00 & 1.00 & 5.00 & 5.00 \\
Agua & 3.09 & 4.85 & 4.03 & ---- & ---- \\
Almidón de maíz & 62.91 & 60.45 & 69.28 & 64.00 & 80.00 \\
\hline & 100 & 100 & 100 & 100 & 100 \\
\hline
\end{tabular}

${ }^{1} \mathrm{TS}$ (torta de soya), HC (harina de carne), HP (harina de pollo), Control (caseína)

tos balanceados comercializados para perros en nuestro medio, provienen de empresas nacionales y extranjeras que utilizan insumos proteicos de diferentes calidades.

Se hizo el análisis proximal a las dietas e insumos proteicos utilizando las técnicas analíticas estandarizadas en el laboratorio (Arbaiza, 1997).

\section{Evaluación biológica en ratas}

Relación de Eficiencia Proteica (PER): Se utilizó 16 ratas machos distribuidas en 4 grupos correspondientes al tipo de dieta administrada (TS, HC, HP y control). Los animales se colocaron en jaulas individuales administrándoles las dietas y el agua ad libitum durante 28 días. La PER se calculó empleando la siguiente expresión:

$$
\text { PER }=\frac{\text { Aumento de peso }(\mathrm{g})}{\text { Consumo }(\mathrm{g}) \times \% \text { Proteína }}
$$

El consumo de alimento se halló restando el peso del alimento suministrado menos el peso del alimento derramado. Se re- gistró el peso corporal de cada rata el primer día y luego cada 7 días hasta el final del periodo de ensayo.

Digestibilidad Verdadera (DV): Se utilizó 20 ratas machos distribuidas en los grupos TS, HC, HP, control y aproteico. Se recolectó las heces, las que fueron secadas y trituradas para determinar el contenido de nitrógeno. La DV se calculó empleando la siguiente fórmula:

$\mathrm{DV}=\underline{\text { Consumo de } \mathrm{N}-(\mathrm{N} \text { fecal }-\mathrm{N} \text { fecal metabólico })}$ Consumo de $\mathrm{N}$

El consumo de $\mathrm{N}$ se halló multiplicando el contenido de $\mathrm{N}(\%)$ por el consumo de alimento (g). El N fecal se halla multiplicando el contenido de nitrógeno (\%) por la cantidad (g) de heces. El N fecal metabólico se estima determinando la cantidad de nitrógeno fecal excretado cuando el animal está consumiendo una dieta libre de proteína.

Utilización Neta de las Proteínas (NPU): Se empleó 10 ratas machos y 10 ratas hembras, distribuidas en los grupos TS, HC, HP, control y aproteico. Se registró los pesos y el 
consumo de alimento. Al final de los 10 días del ensayo se sacrificaron los animales con cloroformo y se secó la carcasa para luego molerla y determinar el contenido de nitrógeno. El valor de NPU se calculó con la siguiente fórmula:

$$
\mathrm{NPU}=\frac{\mathrm{N} \text { muestra }-\mathrm{N} \text { conocido }}{\text { Ingesta de } \mathrm{N}}
$$

Donde:

$$
\begin{aligned}
\mathrm{N} \text { Muestra }= & \text { Contenido de } \mathrm{N} \text { corporal con } \\
& \text { la proteína a probar } \\
\mathrm{N} \text { Conocido }= & \text { Contenido de } \mathrm{N} \text { corporal con } \\
& \text { dieta libre } \mathrm{N}
\end{aligned}
$$

El $\mathrm{N}$ corporal se halló multiplicando el contenido de $\mathrm{N}(\%)$ por el peso de la carcasa (g). La ingesta de $\mathrm{N}$ se halla multiplicando el contenido de $\mathrm{N}(\%)$ por el consumo de alimento $(\mathrm{g})$.

Valor biológico $(\mathrm{VB})$ : Este parámetro se calculó a partir de la fórmula:

$$
\mathrm{VB}=\frac{\mathrm{NPU}}{\mathrm{DV}}
$$

Análisis estadísticos

Se realizó el análisis de varianza para determinar diferencias en el PER de los insumos utilizados y la caseína, y se utilizó la prueba de diferencias mínimas significativas de Fisher para determinar las diferencias entre promedios.

\section{Resultados y Discusión}

Los resultados del análisis proximal de los insumos proteicos y de las dietas experimentales se observan en los cuadros 2 y 3 , respectivamente. Las diferencias entre los porcentajes utilizados en la formulación de las dietas experimentales y los resultados del análisis proximal se debería a variaciones propias del análisis proximal. En el Cuadro 4 se puede observar que los insumos proteicos evaluados mostraron una elevada digestibilidad verdadera.

El valor PER para la caseína fue similar al valor reportado por otros investigadores (Pellet y Young, 1980; Muñoz, 1990). El valor de PER para HC fue significativamente menor al valor de los otros insumos evaluados $(\mathrm{p}<0.05)$. No hubo diferencias significativas entre la PER de la HP y TS (Cuadro 4).

Cuadro 2. Análisis proximal de los insumos proteicos [base húmeda $(\mathrm{BH})$ y base seca $(\mathrm{BS})$ ]

\begin{tabular}{lcccccc}
\hline & \multicolumn{2}{c}{ Dieta TS $^{1}$} & \multicolumn{2}{c}{ Dieta HC $^{1}$} & \multicolumn{2}{c}{ Dieta HP $^{1}$} \\
\cline { 2 - 7 } & BH & BS & BH & BS & BH & BS \\
\hline Humedad (g/100g) & 8.94 & 0.00 & 0.64 & 0.00 & 4.83 & 0.00 \\
Proteína (g/100g) & 46.81 & 51.40 & 43.25 & 43.52 & 49.58 & 52.10 \\
Extracto etéreo (g/100g) & 2.0 & 2.19 & 9.44 & 9.50 & 26.94 & 28.31 \\
Cenizas (g/100g) & 5.11 & 5.61 & 5.38 & 5.41 & 15.11 & 15.68 \\
\hline
\end{tabular}

${ }^{1} \mathrm{TS}$ (torta de soya), HC (harina de carne), HP (harina de pollo) 
Cuadro 3. Análisis proximal de las dietas ${ }^{1}$ [base húmeda $(\mathrm{BH})$ y base seca (BS)]

\begin{tabular}{lcccccccccc}
\hline & \multicolumn{2}{c}{ Dieta TS } & \multicolumn{2}{c}{ Dieta HC } & \multicolumn{2}{c}{ Dieta HP } & \multicolumn{3}{c}{ Dieta control } & \multicolumn{3}{c}{ Dieta aproteica } \\
& BH & BS & BH & BS & BH & BS & BH & BS & BH & BS \\
\hline $\begin{array}{l}\text { Humedad } \\
(\mathrm{g} / 100 \mathrm{~g})\end{array}$ & 8.44 & 0.00 & 8.48 & 0.00 & 8.92 & 0.00 & 4.73 & 0.00 & 5.18 & 0.00 \\
$\begin{array}{l}\text { Proteína } \\
(\mathrm{g} / 100 \mathrm{~g})\end{array}$ & 10.7 & 11.69 & 10.22 & 11.17 & 8.97 & 9.85 & 11.70 & 12.28 & 0.00 & 0.00 \\
$\begin{array}{l}\text { Ext. etéreo } \\
\text { (g/100g) }\end{array}$ & 4.4 & 4.81 & 6.53 & 7.13 & 7.54 & 8.28 & 3.62 & 3.8 & 5.14 & 5.42 \\
$\begin{array}{l}\text { Fibra cruda } \\
(\mathrm{g} / 100 \mathrm{~g})\end{array}$ & 0.31 & 0.34 & 0.20 & 0.22 & 0.17 & 0.19 & 0.88 & 0.92 & 0.25 & 0.26 \\
$\begin{array}{l}\text { Cenizas } \\
\text { (g/100g) }\end{array}$ & 4.81 & 5.25 & 2.76 & 3.02 & 4.52 & 4.96 & 4.45 & 4.67 & 3.36 & 3.54 \\
$\begin{array}{l}\text { Extracto no } \\
\text { nitrogenado } \\
\text { (g/100g) }\end{array}$ & 71.34 & 77.91 & 71.81 & 78.46 & 69.88 & 76.72 & 74.62 & 78.33 & 86.07 & 90.78 \\
\hline
\end{tabular}

${ }^{1} \mathrm{TS}$ (torta de soya), HC (harina de carne), HP (harina de pollo), dieta control (caseína)

Cuadro 4. Resultados de la evaluación biológica de tres insumos proteicos

\begin{tabular}{lcccc}
\hline Insumo & $\mathrm{PER}^{1}$ & $\mathrm{DV}^{2}$ & $\mathrm{NPU}^{3}$ & $\mathrm{VB}^{4}$ \\
\hline Torta de soya & $1.86^{\mathrm{a}}$ & 98.76 & 58.19 & 0.59 \\
Harina de carne & $1.39^{\mathrm{b}}$ & 96.95 & 35.52 & 0.37 \\
Harina de pollo & $1.69^{\mathrm{a}}$ & 98.13 & 60.19 & 0.61 \\
Caseína (Control) & $2.11^{\mathrm{c}}$ & 99.19 & 96.46 & 0.97 \\
\hline
\end{tabular}

$a, b, c$ Letras diferentes dentro de una columna indican diferencias significativas $(p<0.05)$

${ }^{1}$ Relación de eficiencia proteica

2 Digestibilidad verdadera

${ }^{3}$ Utilización neta de las proteínas

${ }^{4}$ Valor biológico

Meller y Bender (Church y Pond, 1990) reportaron valores de NPU para la caseína de 60 , harina de carne de 35.5 a 48.3 y harina de plumas de 21.2 a 35.6. El NPU determinado en el estudio para la HC fue de 35.52 y está dentro del rango propuesto por los autores mencionados; en cambio la caseína tuvo un NPU de 96.46 y fue superior a los valores reportados. Church y Pond (1990) publican VB para la caseína de 0.69 y para la harina de carne entre 0.72 a 0.79 . El VB hallado para la caseína tuvo un valor de 0.97 , muy superior a los valores reportados; en cambio, la HC tuvo el VB de 0.37, muy inferior a los encontrados por estos autores.
Los insumos proteicos evaluados y la caseína tuvieron una DV alto; sin embargo, cuando se realizó las pruebas biológicas (PER, NPU y VB) se pudo apreciar las diferencias en la calidad proteica, siendo la $\mathrm{HC}$ la de menor calidad.

\section{Conclusiones y Recomiendaciones}

- Se determinó que la harina de carne tiene la menor calidad proteica en relación a la torta de soya y la harina de pollo. 
- No basta con determinar el contenido proteico y digestibilidad aparente para evaluar la calidad de los insumos proteicos, sino que es necesario realizar evaluaciones biológicas (PER, NPU y DV).

\section{Literatura Citada}

1. AOAC. Official Methods of Analysis. 1990. Protein Efficiency Ratio (Method 960.48). 15 th. p 1095-1098. Association of Official Analitical Chemist. Virginia, EEUU.

2. Arbaiza, T. 1997. Manual de procedimientos para el análisis proximal de alimentos. Fac. de Medicina Veterinaria, UNMSM. Lima, Perú. 13 p.

3. Case, L.P.; D.P. Carey; D.A. Hirakawa. 1997. Nutrición canina y felina. $2^{\text {da }}$ ed. Ed. Manual para los Profesionales. España. 455 p.

4. Church, D.C.; W.G. Pond. 1990. Fundamentos de nutrición y alimentación de animales. $2^{\text {da }}$ ed. Ed. Limusa. México. $438 \mathrm{p}$.

5. Fataccioli, D. 1981. Evaluación biológica de la harina de plumas en ratas y ovinos. Tesis Bachillerato. Fac Zootecnia, UNALM. Lima, Perú. 63 p.

6. Muñoz, A. 1990. Alimentación y nutrición. Ed. Ediagraria-UNALM. Consejo Nacional de Ciencia y Tecnología del Perú. p 60-62.

7. Pellet, P.; V. Young. 1980. Evaluación nutricional de los alimentos proteínicos. Universidad de las Naciones Unidas. Tokyo, Japón. 174 p.

8. Piccioni, H. 1970. Diccionario de alimentación animal. $3^{\text {ra }}$ ed. Ed. Acribia. Zaragoza, España. 819 p. 